\title{
Positive Predictive Value of Blood Cultures Utilized by Community Emergency Physicians
}

\author{
Edward C. Lin, ${ }^{1}$ and Kevin M. Boehm ${ }^{1,2,3}$ \\ ${ }^{1}$ Department of Emergency Medicine, Henry Ford Wyandotte Hospital, Wyandotte, MI 48192, USA \\ ${ }^{2}$ College of Osteopathic Medicine, Michigan State University, East Lansing, MI 48824, USA \\ ${ }^{3}$ College of Osteopathic Medicine, Nova Southeastern University, Fort Lauderdate, FL 33314, USA
}

Correspondence should be addressed to Kevin M. Boehm; kboehm1@hfhs.org

Received 26 June 2012; Accepted 7 August 2012

Academic Editors: Y.-H. Gan, T. Matsumoto, T. A. Rupprecht, and G. Simo

\begin{abstract}
Copyright (C) 2013 E. C. Lin and K. M. Boehm. This is an open access article distributed under the Creative Commons Attribution License, which permits unrestricted use, distribution, and reproduction in any medium, provided the original work is properly cited.

Objective. To determine the positive predictive value of blood cultures obtained from patients from a suburban ED and how the ED physicians use empirical antibiotics. Method. This retrospective chart review study was conducted at the ED of a suburban teaching hospital. The study consisted of adult patients who presented to the ED with evidence of clinical conditions suggesting bacteremia. Result. Over a 12-month period, 408 patients with positive blood cultures were drawn in the ED. The mean age of the patients was 65.85 years. The positive predictive value was $58.3 \%$, and community ED physicians used appropriate empirical antibiotics in $72.3 \%$ of patients with true bacteremia. Conclusion. The positive predictive value indicated that many of the positive blood culture results were false positives from skin contamination. Blood cultures are necessary to follow up any positive results with more reliable tests to obtain a more accurate assessment as to whether bacteremia is present in the emergency department. True positive blood cultures can assist other doctors in adjusting the antibiotics. Emergency physicians overall used appropriate antibiotics in $72.3 \%$ of patients with true bacteremia. This study demonstrated that emergency physicians have the potential to improve the empirical treatment in treating patients.
\end{abstract}

\section{Introduction}

Each year, 123.8 million people visit emergency departments across the United States [1]. Febrile episodes are one of the conditions most commonly seen in emergency departments. Examples of such episodes include pneumonia, sepsis, mental status change, trauma, being immunocompromised, leukocytosis, febrile leukopenia, or a history of IV drug use. Emergency physicians order blood cultures when patients are suspect for various febrile episodes. Additionally, the Joint Commission (previously the Joint Commission on the Accreditation of Healthcare Organizations or JCAHO) requires blood cultures to be performed in the emergency department prior to initial antibiotics received in the emergency department for hospitalized community acquired pneumonia (CAP) patients [2, 3]. Despite its limitation, the blood culture remains the "gold standard" for the detection of bacteremia. Blood cultures are important for defining local spectra of pathogens and resistance in severe infections. Although blood cultures are ordered, the results often are not obtained until after initial treatment for the patient has begun. After seeing the patient, ER physicians select an antibiotic to treat the patient before the culture results have been obtained. The purpose of this study is to utilize the electronic medical record systems of a community-based, academic hospital to investigate the positive predictive value of blood cultures in the local community ED and whether community ED physicians selected appropriate antibiotics for the initial treatment compared with the culture results.

\section{Patients and Methods}

2.1. Study Design. We conducted a retrospective chart review study on adult population visits to the emergency department with blood culture diagnostic workup during one calendar 
year (from January 2009 to December 2009). The suburban community hospital has a total of 344 beds within the hospital and 49 beds in the emergency department. The emergency department provides care for approximately 66,000 patients per year. $27 \%$ of the ED adult patients are subsequently admitted to the hospital, and $0.88 \%$ of the patients are admitted to critical care units.

The emergency department is staffed by emergency medicine board certified attending physicians and emergency medicine resident physicians. All resident physicians are directly supervised by EM faculty attending physicians.

The study was performed in accordance with the rules of the observational studies by the Henry Ford research administration committee. Patients' informed consents were not required since this was a retrospective chart review process. The blood cultures were done for clinical purposes and were conducted according to accepted clinical standard.

During the study period, ED residents or attending physicians ordered a pair of blood cultures. A pair of blood cultures consists of one aerobic bottle and one anaerobic bottle. Prior to venipuncture, the patient's skin was prepped with ChloraPrep for 2 minutes. Blood was drawn via sterile technique, and 5-10 mL of blood was transferred into an aerobic and an anaerobic culture media vial. ED staff waited approximately 15-20 minutes to draw the second set of blood cultures from a different peripheral site. There were a small number of patients from whom the staffs were unable to obtain peripheral blood. Therefore, only 1 set of blood cultures was obtained through central line in a sterile setting.

After blood was extracted for the culture, the media vials were incubated at $35^{\circ} \mathrm{C}$ in the microbiology laboratory. Aerobic vials were placed on a rotary shaker. An automated blood culture instrument (versa-trek) was used to detect microbial growth by monitoring changes in barometric pressure. Bottles were tested on a regular basis automatically by the instrument. Any change in barometric pressure signaled a positive culture and was subcultured on appropriate media. The mean time for development of positive blood cultures was 8-48 hours. All blood culture bottles were updated on a daily basis, and in five days, they were finalized. Microbiology lab followed Clinical and Laboratory Standard Institute (CLSI) and Henry Ford Wyandotte Hospital pharmaceutical formulary for susceptibility testing.

2.2. Data Collection. The microbiology department performs periodic quality assurance studies specifically addressing ED blood culture contamination rates. Only the results of the first two blood cultures drawn on the date of ED visits are considered. The clinical relevance of bacteria commonly found in the skin flora isolated in blood culture is often difficult to determine. In our study, coagulase-negative staphylococci, Streptococcus viridans, Corynebacterium spp., Bacillus spp., Micrococcus spp., Peptostreptococcus spp., Propionibacterium spp., Aerococcus species, Acinetobacter iwoffi, Clostridium perfringens, and aerobic spore forming gram positive rods were termed "skin flora" and considered contaminating [47]. Isolates of coagulase negative staphylococci and alpha hemolytic streptococci were considered pathogenic if they were isolated from two or more blood cultures bottles and if the following conditions were met: being immunocompromised, whether an intravascular catheter or prosthetic device was present, and whether there was a history of IV drug abuse [6]. All other isolated pathogens were considered obligate pathogens.

For patients with positive blood culture results, a detailed review of the emergency medical record (IBEX) was undertaken to document patient's age, medical record number, ED attending name, the chief complaint, empirical antibiotic selection by the emergency physicians, immunocompromised status, any recent surgery, presence of new prosthesis insertion, history of IV drug abuse, and ED diagnosis. Chart review of positive blood cultures was performed. Organisms based upon microbiology department results updated in CarePlus were also documented. Microbiology labs also provided susceptibility result published in CarePlus if pathogens were identified.

The empiric antimicrobial therapy was considered effective when the organism was susceptible to one or more of the antibiotics prescribed based on microbiology lab susceptibility results or antibiogram published by Henry Ford Wyandotte hospital. When the antibiotics chosen by emergency physician were not listed by the microbiology department susceptibility results, "Sanford Guide to Antimicrobial Therapy" was then utilized to determine susceptibility. The Sanford Guide considers antibiotics to be susceptible when they are usually effective clinically or $>60 \%$ susceptible [8].

2.3. Variable and Definitions. The variables obtained in the ED included age, symptoms (fever and shivering), neoplasm, IV drug use, immunosuppression, main diagnosis from ED, identification of the organism, and antibiotic treatment given during stay in the ED.

2.4. Statistical Analysis. The data was analyzed by the statistical department at Henry Ford Health System. All analyses were performed using SAS 9.2. There were a total of 410 positive blood cultures included in the data set. Two patients were excluded because no ED chart was found, leaving a sample size of $N=408$ positive blood cultures. Positive predictive value is calculated by the number of true positives divided by the total number of positive blood cultures. The mean, median, standard deviation, minimum, and maximum are included for the continuous variable age. For categorical variables, the frequency and percent are reported. Percentages were based on the sample size $N=408$, unless otherwise noted.

\section{Result}

During the 12-month period from January to December 2009, Henry Ford Wyandotte emergency department physicians ordered total 9,791 sets of blood cultures. 25 sets of positive pediatric blood cultures were excluded from the additional analysis. Of these total sets of blood cultures, 408 sets were positive cultures from adult. 238 sets yielded 
pathological bacteremia and 170 sets were classified as contamination with skin flora. Therefore, the positive predictive value was $58.33 \%$. Gram negative organisms were isolated in 96 sets and gram positive organisms in 132.

Patients with positive blood culture results were predominantly elderly (age 18-97, with mean 65.85, median 67). $50 \%$ of the sample is older than 67 years old (Table 1). 16 patients with recent line insertion/surgical procedure performed (3.92\%), 17 patients were on dialysis (4.17\%). Emergency physicians order blood cultures when patients presented with fever, were immunocompromised, or suspect for pneumonia, sepsis, extensive cellulitis, mental status change, trauma, leukocytosis, leukopenia, or a history of IV drug use/recent surgical procedure. The main ED diagnoses from positive blood cultures were sepsis, pneumonia, urosepsis, pyelonephritis, suspected line/port infection, and cellulitis.

The majority of microorganism from positive blood cultures $(N=408)$ were MRSA $(9.31 \%)$, E. coli $(12.99 \%)$ Streptococcus pneumoniae (3.92\%), Klebsiella pneumonia (2.94\%), Proteus mirabilis (1.72\%), Pseudomonas species (2.45\%), Enterobacter (0.98\%), and candidiasis (1.72\%). The majority of skin contamination were Staphylococcus coagulase negative (59.41\%), Corynebacterium sp. (13.53\%), Peptostreptococcus spp. (1.18\%), Bacillus sp. (2.94\%), and alpha Streptococcus (5.88\%).

Patients who were diagnosed with pneumonia $(N=131)$, the most likely microorganisms were Streptococcus pneumoniae (11.45\%), MRSA (8.40\%), Klebsiella pneumonia (4.58\%), and E. coli (7.63\%) Patients who were diagnosed with urosepsis/pyelonephritis $(N=9.62)$, the most likely pathogen were E. coli (32.29\%) and Proteus mirabilis (6.25\%). The most likely pathogen isolated from blood cultures for extensive cellulitis/decubitus ulcer $(N=52)$ was MRSA (9.62\%).

The most commonly used antibiotics were vancomycin (46.08\%), followed by ciprofloxacin/levofloxacin $(29.90 \%)$ and piperacillin/tazobactam (23.77\%) (See Table 2 for other emperic antibiotics selected). The empirical antimicrobial therapy was considered effective in $72.27 \%$ of the time in the emergency department (172 out of 238). Of the remaining 66 patients $(27.73 \%)$, emergency physicians used inappropriate antibiotics in 29 cases $(12.18 \%)$ where there is no susceptibility match. Emergency physicians did not prescribe antibiotics in 20 patients $(8.4 \%)$ with true positive blood cultures result. There were 16 patients $(24.2 \%, N=66)$ (with recent hospital admission due to surgery/dialysis) with Staphylococcus infection, and ED physicians did not cover MRSA with vancomycin. There were 11 patients (4.6\%) with positive blood culture and sensitivity tests showed resistance to the antibiotics prescribed by emergency physicians. Six patients $(2.52 \%)$ with fungemia and emergency physician failed to cover with antifungal medications. Overall, no empirical antibiotic therapy was started in only 64 episodes (15.69\%) after blood culture drawn in the $\operatorname{ED}(N=408)$.

\section{Discussion}

Blood cultures were useful for patients who are febrile and appear ill. Identifying emergency department patients with
TABLE 1: Descriptive statistics for age.

\begin{tabular}{ccccccc}
\hline & Mean & Median & Std. Dev. & Min. & Max. & $N$ \\
\hline Age & 65.85 & 67 & 17.19 & 18 & 97 & 408 \\
\hline
\end{tabular}

TABLE 2: Empiric antibiotic selection.

\begin{tabular}{lcc}
\hline Antibiotic & Frequency & Percent \\
\hline Metronidazole & 13 & $3.19 \%$ \\
Azithromycin & 34 & $8.33 \%$ \\
Ciprofloxacin/levofloxacin & 122 & $29.90 \%$ \\
Doxycycline & 2 & $0.49 \%$ \\
Vancomycin & 188 & $46.08 \%$ \\
Ceftriaxone/ceftazidime & 79 & $19.36 \%$ \\
Gentamycin/tobramycin & 43 & $10.54 \%$ \\
Piperacillin and tazobactam & 97 & $23.77 \%$ \\
Ampicillin & 5 & $1.23 \%$ \\
Ampicillin and Sulbactam & 19 & $4.66 \%$ \\
Clindamycin & 10 & $2.45 \%$ \\
Moxifloxacin & 17 & $4.17 \%$ \\
Aztreonam & 25 & $6.13 \%$ \\
Imipenem & 1 & $0.25 \%$ \\
Cefepime & 29 & $7.11 \%$ \\
Sulfamethoxazole and trimethoprim & 3 & $0.74 \%$ \\
Specialty micafungin acyclovir & 3 & $0.74 \%$ \\
Linezolid & 2 & $0.49 \%$ \\
Cefazolin & 3 & $0.74 \%$ \\
Tigecycline & 1 & $0.25 \%$ \\
\hline
\end{tabular}

bacteremia presented an important and difficult challenge for emergency clinicians. In patients with bacteremia, the symptoms and signs were sometimes subtle, such as altered mental status. Fortunately, with early goal-directed therapy, emergency physicians were aggressive to order blood cultures and started empirical antibiotic therapies early in the emergency department. Our hospital also had antibiotic bundles for patients who presented with sepsis based on the most likely source of infection. Negative blood cultures results rarely changed the management of medical inpatients. For example, emergency physicians still order antibiotics for patients suspecting pneumonia (either hospital/community acquired) with negative blood cultures. While good followup is imperative, positive blood cultures in this setting may help guide the treating physicians in the inpatient management.

It was challenging to differentiate contamination from true bacteremia. Clues such as identity of organism, number of positive culture sets, number of positive bottles within a set, time growth, quantity of growth, clinical and laboratory data, and source of culture can help clinicians to differentiate contamination from bacteremia. The College of American Pathologists (CAP) Q-Probes study found that the most important indicator when interpreting blood culture result was the identity of the organism [9]. Based on that study, we distinct obligate pathogen and contamination based on the species of bacteria in our study. However, it could be also some possible flaws because some of the 
alpha streptococcal isolated grouped as pathogen could be contaminants. While the isolated coagulase negative staphylococci groups as contamination may have represented the actual pathogen responsible for the infection [4]. As results, we also considered patients with prosthetic device [4], recent surgery, immunosuppression (HIV or cancer patients receiving chemo/radiation therapy) $[5,7]$, intravascular catheter $[4,7]$, and history of drug addiction [5]. Similar criteria have been previously used by others [10].

It has been the practice in our emergency department to obtain two sets of blood cultures from different sites per adult patients. More than two blood culture specimen improve the interpretation of blood culture results. During the study period, the standard for performing blood cultures in the emergency department was to obtain blood by two separate venipunctures [11]. Unfortunately, the staff of the ED did not adhere to this standard. From the author's observation, ED staff commonly drew 2 sets of blood cultures from the same site of venipuncture, rather than 2 separate sites. This is especially true for patients on hemodialysis or for patients with low blood pressure and other malignancies because of convenience and reduction of trauma to veins [11]. Since this is the retrospective chart review, the prospective study could eliminate contamination problems by following the standard protocols. If impractical to draw cultures at two separate sites, perhaps future studies on culturing the skin prior to prepping for venipuncture would be helpful in sorting out true bacteremia from skin contamination.

The empirical antibiotic therapy susceptibility was lower than what we expected, especially since the hospital adopted early goal-directed therapy. The most likely explanation was emergency physicians overlooked the fact that patients presented with dialysis catheter or bacteremia due to risk of recent surgery. This resulted in emergency physicians failing to cover MRSA infection. For instance, there were 16 patients $(24.2 \%, N=66)$ (with recent hospital admission due to surgery/dialysis) with Staphylococcus infection, and ED physicians fail to cover MRSA with vancomycin. Another possible reason was the resistance of pathogen to the empirical antibiotics prescribed by the emergency physicians. We recommended emergency physician to spend additional time investigating previous antibiotic resistance of pathogen from patient's previous microbiology result. Finally, there were 64 cases $(15.69 \%)$ where ED physician ordered blood cultures but no antibiotic documented. Looking at the final diagnoses from emergency department, the majority of emergency physicians thought the causes of the symptoms were less likely infectious.

We were unable to identify patients with false negative blood cultures because data for negative blood cultures were not kept on file. However, given the low prevalence of true positive cultures, we believe that the number of false negatives would be small [2]. Since negative blood cultures are not used as a basis of clinical decision making in the emergency department, it is unlikely that false negative cultures (for patients who still had symptoms consistent with bacteremia) would have had an impact on morbidity [5].

\section{Conclusion}

The result of the positive predictive value ( $\mathrm{PPV}=58.3 \%)$ indicated that many of the positive blood culture results were false positive from skin contamination. Blood culture is neither a high-yield nor low-yield test. Thus, it is necessary to follow up any positive results with a more reliable test (such as repeated blood cultures inpatient, clinical exam findings, and vital signs) to obtain a more accurate assessment as to whether bacteremia is present in the emergency department. However, true positive blood cultures can assist other medical doctors (infectious disease and internal medicine doctors) in adjusting the antibiotics (addition or deescalate). Community emergency physicians at our institution overall used appropriate antibiotics in $72.3 \%$ of patients with true bacteremia. This study demonstrated that community emergency physicians have the potential to improve the empirical antibiotic treatment in treating critical ill patients and to provide stabilization for patients with bacteremia.

\section{References}

[1] Center for Disease Control and Prevention, http://www.cdc .gov/nchs/fastats/hospital.htm.

[2] Joint Commission on Accreditation of Healthcare Organizations, "A comprehensive review of development and testing for national implementation of hospital core measures," http://www.jointcommission.org/specifications_manual_for_ national_hospital_inpatient_quality_measures.aspx.

[3] Joint Commission on Accreditation of Healthcare Organizations, NHQM Specification Manual for National Quality Measures, Measure Information Form, Version 2.1, http://www.jointcommission.org/specifications_manual_for_ national_hospital_inpatient_quality_measures.aspx.

[4] K. K. Hall and J. A. Lyman, "Updated review of blood culture contamination," Clinical Microbiology Reviews, vol. 19, no. 4, pp. 788-802, 2006.

[5] K. M. Sturmann, J. Bopp, D. Molinari, S. Akhtar, and J. Murphy, "Blood cultures in adult patients released from an urban emergency department: a 15-month experience," Academic Emergency Medicine, vol. 3, no. 8, pp. 768-775, 1996.

[6] M. P. Weinstein, "Current blood culture methods and systems: clinical concepts, technology, and interpretation of results," Clinical Infectious Diseases, vol. 23, no. 1, pp. 40-46, 1996.

[7] J. M. - Ramos, M. Masia, M. Elia et al., "Epidemiological and clinical characteristics of occult bacteremia in an adult emergency department in Spain: influence of blood culture results on changes in initial diagnosis and empiric antibiotic treatment," European Journal of Clinical Microbiology and Infectious Diseases, vol. 23, no. 12, pp. 881-887, 2004.

[8] D. - Gilbert, R. C. Moellering, G. M. Eliopoulos, and M. A. Sande, The Sandord Guide to Antimicrobial Therapy, vol. 37, Antimicrobial Therapy, 2007.

[9] D. W. Bates and T. H. Lee, "Rapid classification of positive blood cultures: prospective validation of a multivariate algorithm," The Journal of the American Medical Association, vol. 267, no. 14, pp. 1962-1966, 1992. 
[10] D. P. Sklar and R. Rusnak, "The value of outpatient blood cultures in the emergency department," American Journal of Emergency Medicine, vol. 5, no. 2, pp. 95-100, 1987.

[11] R. R. Magadia and M. P. Weinstein, "Laboratory diagnosis of bacteremia and fungemia," Infectious Disease Clinics of North America, vol. 15, no. 4, pp. 1009-1024, 2001. 


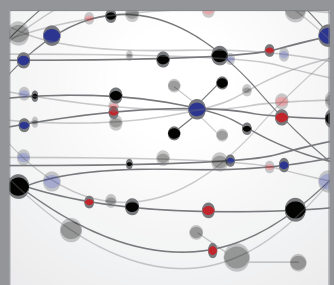

The Scientific World Journal
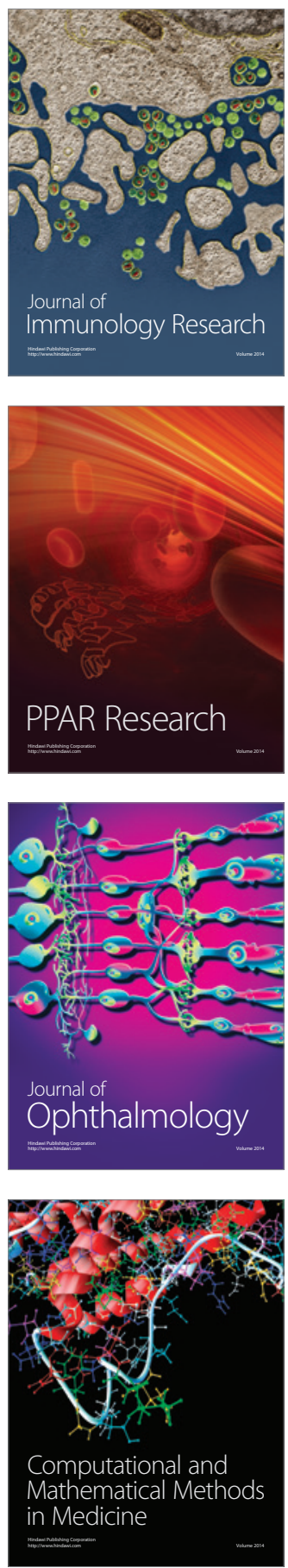

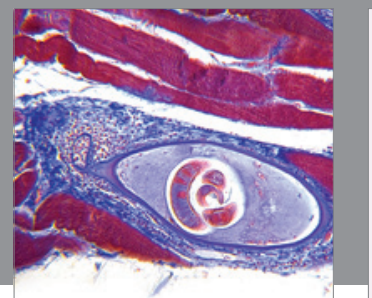

Gastroenterology

Research and Practice
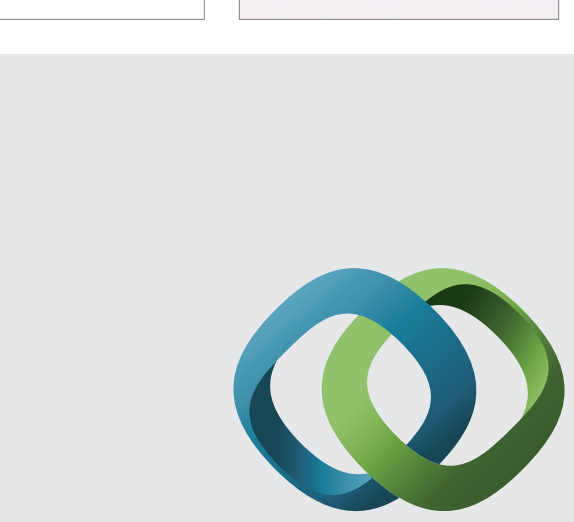

\section{Hindawi}

Submit your manuscripts at

http://www.hindawi.com
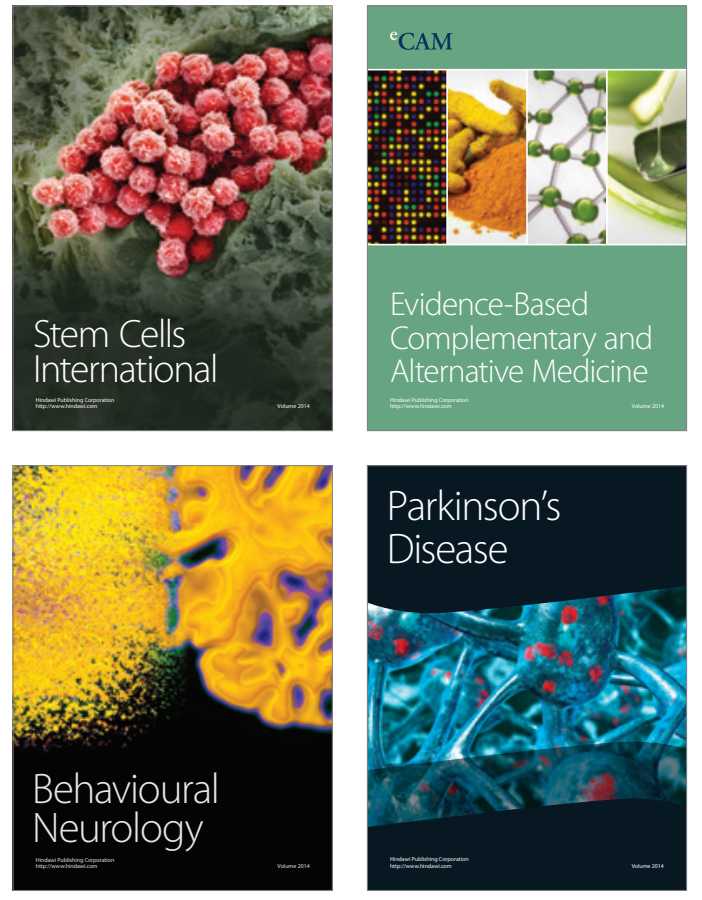
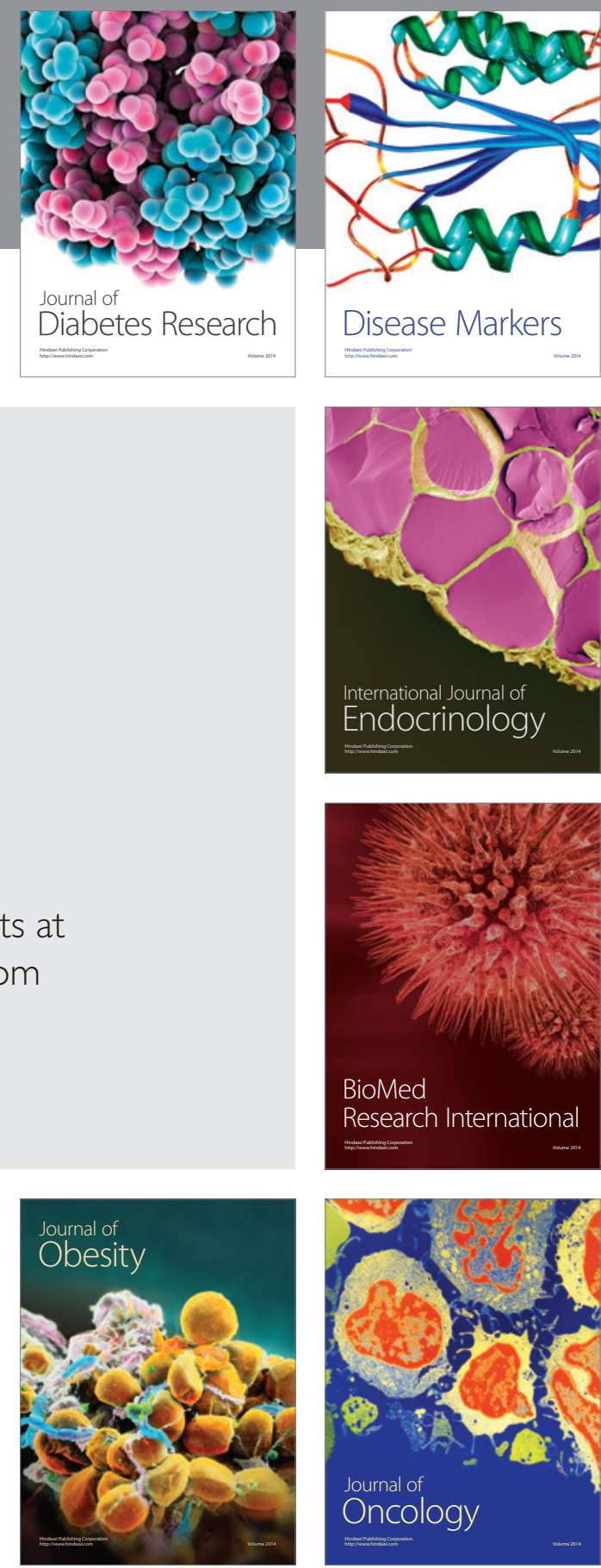

Disease Markers
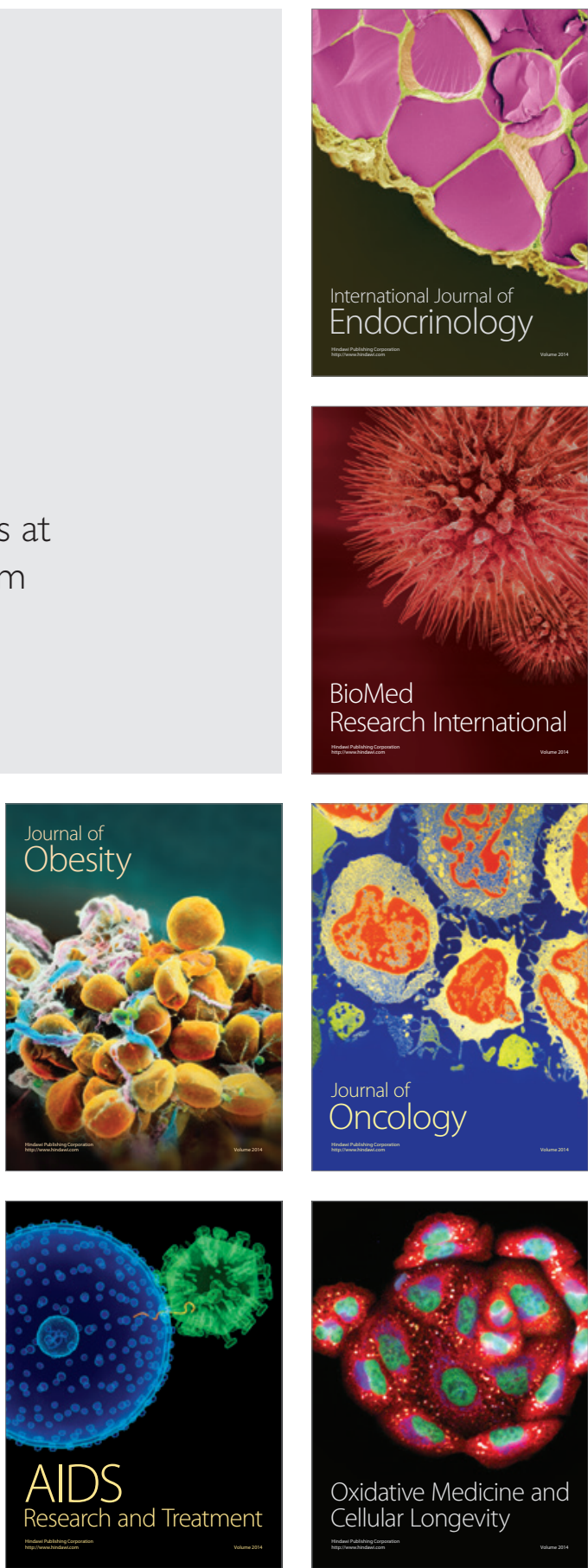